\title{
Genetic and Phenotypic Variabilities of Gerbera (Gerbera jamesonii H. Bolus) Generated from Hybridization
}

\author{
Kurnia Yuniarto $^{1}$, Rika Meilasari ${ }^{2 *}$ and Ridho Kurniati ${ }^{1}$ \\ ${ }^{I}$ Indonesian Ornamental Crop Research Institute, \\ Jl. Raya Pacet- Ciherang Cianjur West Java - Indonesia 43253, PO.BOX 8 SDL \\ ${ }^{2}$ West Sumatera Assessment Institute for Agricultural Technology, \\ Jl. Raya Padang Solok Km.40 Sukarami, Kab. Solok. West Sumatera, Indonesia. PO Box 34 Padang \\ *Corresponding author.Email: rimeisa@yahoo.com
}

\begin{abstract}
The novelty and superior new varieties of gerbera were required in floriculture industries. There were obtained by hybridization to produce variabilities in genotypic and phenotypic characters. The aim of the study was to describe genetic and phenotypic variabilities of 10 varieties of gerbera from hybridization. The study was conducted in Indonesian Ornamental Crops Research Institute, from March until August 2017. Design of the research was Random Completely Block Design. Observations were made for both quantitative and qualitative characters. The quantitative characters were consisted of leaf length, leaf width, petiole length, peduncle length, peduncle diameter, flower head diameter, diameter of first inner ray florets, disc floret diameter, outer ray floret length, outer ray floret width, and height of involucre flower head. Qualitative characters were consisted of intensity of anthocyanin coloration at base peduncle, anthocyanin coloration at top peduncle, flower head type, anthocyanin coloration at distal part of inner bracts flower head, outer ray floret shape. Other characters observed were profile in cross section of middle part of ray at outer ray floret, color of inner side at outer ray floret, number of color of outer ray floret, dark disc (before opening of disc floret), main color of perianth lobes at disc floret of outer rows, and color of top relative to other parts of pappus. The result indicated that value of genetic variabilities was high for flower head diameter, diameter of first inner ray florets, disc floret diameter, outer ray floret length, and outer ray floret width. Phenotypic variability value was high for all characters. The heritability value was about $1.47 \%$ until $94.63 \%$. The heritability value was high for leaves width, petiole length, flower head diameter, diameter of first inner ray florets, disc floret diameter, outer ray floret length and outer ray floret width. While, the moderate heritability was obtained for peduncle length and height of involucre flower head. Characters obtained with low heritabilitywere leaves length and peduncle diameter.
\end{abstract}

Keywords: Gerbera, Phenotypic, Genetic variability, Heritability

\section{INTRODUCTION}

Gerbera (Gerbera jamesonii $\mathrm{H}$ Bolus) is popular ornamental plant for cutting flower. Most of cutting plant gerbera used as decoration and bouquet. Gerbera was combined into other flower such as rose, orchids, and snapdragon [1]. Gerbera is perennial herbs, belonging into Asteraceae (Composite). These herbs distributed from South of Africa, Madagascar to East of Asia [2], [3]. These here are stemless and hairy throughout, with foliage arranged in the form of a rosette at the base. Gerbera's leaves are radially arranged, petiolate, lanceolate, deeply lobed, sometimes leathery, narrower at the base and wider at the tip. The flower, known as head or capitulum has conspicuous ray florets in one or two whorls. The flowers are daisy like, there are $7-10 \mathrm{~cm}$ across and extend upto $10-15 \mathrm{~cm}$ in some hybrids. Flowers are in wide range of colors like yellow, orange, cream, white, pink, brick red, scarlet, salmon, maroon and in various intermediate shades [4].

In the past, Gerbera was cultivated in intercropping because it has no fragrance and wither inflorescence. At present, the hybrids of Gerbera could substitute daisy and chrysanthemum as they have bigger flower $(10 \mathrm{~cm})$ and wide range of colors [5]. Indonesian Ministry of Agriculture [6] noted that production of Gerbera increased 14.751 .610 stalks (2017) to 26.608 .911 stalks (2018).

Breeding of Gerbera in Indonesian Ornamental Crops Research conducted since 2012 until now. The objective of gerbera's breeding program were to find out of genetic variability based on morphological characters and their performance. 


\section{MATERIALS AND METHOD}

The research conducted in Indonesian Ornamental Crops Research Institute, March to August 2017. The experiment using Random Completely Block Design (RCBD). Ten varieties gerbera were used as material. The varieties were Athalia Agrihorti, Zsofia Agrihorti, Ayudia Agrihorti, Neoma Agrihorti, Nashita Agrihorti, Nirwasita Agrihorti, Arkadewi Agrihorti, Nalini Agrihorti, Kinandari Agrihorti and Candramaya Agrihorti. The observations were made for leaf length, leaf width, petiole length, peduncle length, peduncle diameter, flower head diameter, diameter of first inner ray florets, disc floret diameter, outer ray floret length, outer ray floret width, and height of involucre flower head. Qualitative characters were intensity of anthocyanin coloration at base peduncle, anthocyanin coloration at top peduncle, flower head type, anthocyanin coloration at distal part of inner bracts flower head, outer ray floret shape. Other qualitative characters were profile in cross section of middle part of ray at outer ray floret, color of inner side at outer ray floret, number of color of outer ray floret, dark disc (before opening of disc floret), main color of perianth lobes at disc floret of outer rows, and color of top relative to other parts of pappus. The formula based on Anderson \& Brancroft [7] was used to estimate the genetic and phenotypic variances:

$$
\begin{aligned}
& \sigma_{\mathrm{g}}^{2}=\left(\mathrm{MS}_{\text {genotype }}-\mathrm{MS}_{\text {error }}\right) / \mathrm{r} ; \sigma_{\mathrm{f}}^{2}=\sigma_{\mathrm{g}}^{2}+\mathrm{MS}_{\text {error }} \\
& \sigma_{\sigma_{g}^{2}}=\sqrt{\frac{2}{r^{2}}\left\{\frac{M S_{\text {genotype }}^{2}}{d b \text { genotype }+2}+\frac{M S_{\text {error }}^{2}}{d b \text { error }+2}\right\}} \\
& \sigma_{\sigma_{f}^{2}}=\sqrt{\frac{2}{r^{2}}\left\{\frac{M S_{\text {genotype }}^{2}}{d b \text { genotype }+2}\right\}}
\end{aligned}
$$

Genetic variance is categorized as broad if $\sigma_{\mathrm{g}}^{2} /$ $\sigma_{\sigma_{g}^{2}}>2$ and phenotypic variance is also categorized as broad if $\sigma_{\mathrm{f}}^{2} / \sigma_{\sigma_{f}^{2}}>2.00[7]$. Heritability was estimated based on Fehr [8]

$$
h^{2}=\frac{\sigma_{g}^{2}}{\sigma_{f}^{2}} \times 100 \%
$$

Heritability estimate was classified based on Mangoendidjojo [9], namely high $\left(\mathrm{h}^{2}>50 \%\right)$, medium $\left(20 \%<\mathrm{h}^{2}<50 \%\right)$, and low $\left(\mathrm{h}^{2}<20 \%\right)$.

\section{RESULTS AND DISCUSSION}

Broad genetic variability were obtained for flower head diameter, diameter of first inner ray florets, disc floret diameter, outer ray floret length and outer ray floret width. Narrow genetic variability were obtained for leaf length, leaf width, petiole length, peduncle length, peduncle diameter and height of involucre flower head. Phenotypic variability was found to be broad for all parameters observed.

Heritability estimates ranged from $1.47 \%$ to $94.63 \%$. High heritability was obtained for leaf width, petiole length, flower head diameter, diameter of first inner ray florets, disc floret diameter, outer ray floret length, and outer ray floret width.

Medium heritability was obtained for peduncle length and height of involucre flower head, while low heritability obtained by leaf length and peduncle diameter. Highest heritability was obtained for diameter of first inner ray florets, while lowest heritability was obtained by peduncle diameter (Table $1)$.

Table 1. Genetic parameters estimated from ten varieties Gerbera.

\begin{tabular}{|c|l|l|l|l|l|l|l|l|l|}
\hline No & \multicolumn{1}{|c|}{ Parameter Observed } & $\sigma_{\mathrm{g}}^{2}$ & $\sigma_{\sigma_{\mathrm{g}}^{2}}$ & $\begin{array}{c}\text { Type of } \\
\text { genetic } \\
\text { variability }\end{array}$ & $\sigma_{\mathrm{f}}^{2}$ & $\sigma_{\sigma_{f}^{2}}$ & $\begin{array}{c}\text { Type of } \\
\text { phenotypic } \\
\text { variability }\end{array}$ & $\begin{array}{c}\mathbf{h} \\
(\%)\end{array}$ & h type \\
\hline 1 & Leaf length & 6.461 & 8.704 & narrow & 42.263 & 7.844 & broad & 15.29 & low \\
\hline 2 & Leaf width & 10.786 & 5.989 & narrow & 20.004 & 5.909 & broad & 53.92 & high \\
\hline 3 & Petiole length & 5.645 & 2.899 & narrow & 8.956 & 2.878 & broad & 63.03 & high \\
\hline 4 & Peduncle length & 58.359 & 35.225 & narrow & 125.984 & 34.496 & broad & 46.32 & medium \\
\hline 5 & Peduncle diameter & 0.015 & 0.179 & narrow & 1.000 & 0.146 & broad & 1.47 & low \\
\hline 6 & Flower head diameter & 0.753 & 0.364 & broad & 1.048 & 0.363 & broad & 71.85 & high \\
\hline 7 & Diameter of first inner ray florets & 2.852 & 1.239 & broad & 3.014 & 1.239 & broad & 94.63 & high \\
\hline 8 & Disc floret diameter & 0.598 & 0.280 & broad & 0.768 & 0.279 & broad & 77.86 & high \\
\hline 9 & Outer ray floret length & 0.062 & 0.028 & broad & 0.075 & 0.028 & broad & 82.67 & high \\
\hline 10 & Outer ray floret width & 0.031 & 0.013 & broad & 0.033 & 0.013 & broad & 93.88 & high \\
\hline 11 & Height of involucre flower head & 0.118 & 0.075 & narrow & 0.276 & 0.073 & broad & 42.75 & medium \\
\hline
\end{tabular}


Table 2. Qualitative Character of ten varieties of Gerbera

\begin{tabular}{|c|c|c|c|c|c|c|c|c|c|c|c|}
\hline No & Character & ATH & ZSO & AYU & NEO & NAS & NIR & ARK & NAL & KIN & CAN \\
\hline 1 & $\begin{array}{l}\text { Peduncle: } \\
\text { Intensity of } \\
\text { anthocyanin } \\
\text { on } \\
\text { coloration } \\
\text { at base } \\
\end{array}$ & weak & weak & moderate & moderate & strong & strong & weak & weak & moderate & strong \\
\hline 2 & $\begin{array}{l}\text { Peduncle: } \\
\text { anthocyanin } \\
\text { coloration } \\
\text { at top }\end{array}$ & present & present & present & present & present & present & absent & absent & present & absent \\
\hline 3 & $\begin{array}{l}\text { Flower head } \\
\text { type }\end{array}$ & $\begin{array}{l}\text { Semi } \\
\text { double }\end{array}$ & $\begin{array}{l}\text { Semi } \\
\text { double }\end{array}$ & $\begin{array}{l}\text { Semi } \\
\text { double }\end{array}$ & Semi double & $\begin{array}{l}\text { Semi } \\
\text { double }\end{array}$ & $\begin{array}{l}\text { Semi } \\
\text { double }\end{array}$ & $\begin{array}{l}\text { Semi } \\
\text { double }\end{array}$ & double & single & $\begin{array}{l}\text { Semi } \\
\text { double }\end{array}$ \\
\hline 4 & $\begin{array}{l}\text { Flower: } \\
\text { anthocyanin } \\
\text { in basal } \\
\text { inner } \\
\text { bractea }\end{array}$ & present & present & present & absent & present & present & present & absent & present & present \\
\hline 5 & $\begin{array}{l}\text { Outer ray } \\
\text { floret: shape }\end{array}$ & $\begin{array}{l}\text { narrow } \\
\text { elliptic }\end{array}$ & $\begin{array}{l}\text { narrow } \\
\text { elliptic }\end{array}$ & $\begin{array}{l}\text { narrow } \\
\text { elliptic }\end{array}$ & narrowobovate & $\begin{array}{l}\text { narrow } \\
\text { obovate }\end{array}$ & $\begin{array}{l}\text { narrow } \\
\text { obovate }\end{array}$ & $\begin{array}{l}\text { narrow } \\
\text { elliptic }\end{array}$ & $\begin{array}{l}\text { narrow } \\
\text { elliptic }\end{array}$ & $\begin{array}{l}\text { narrow } \\
\text { obovate }\end{array}$ & $\begin{array}{l}\text { narrow } \\
\text { obovate }\end{array}$ \\
\hline 6 & $\begin{array}{l}\text { Outer ray } \\
\text { floret : } \\
\text { profile in } \\
\text { cross } \\
\text { section of } \\
\text { middle part } \\
\text { of ray }\end{array}$ & convex & convex & straight & convex & convex & straight & straight & straight & straight & convex \\
\hline 7 & $\begin{array}{l}\text { Outer ray } \\
\text { floret : } \\
\text { Inner color }\end{array}$ & $\begin{array}{l}\text { Red } \\
\text { Purple } \\
\text { Group } \\
62 \mathrm{~A}\end{array}$ & $\begin{array}{l}\text { Green } \\
\text { White } \\
\text { Group } \\
157 \text { C }\end{array}$ & $\begin{array}{l}\text { Red } \\
\text { Group } \\
55 \mathrm{D}\end{array}$ & $\begin{array}{l}\text { End ofpetal: } \\
\text { Yellow } \\
\text { Group 6A; } \\
\text { Middle of } \\
\text { petal: Orange } \\
\text { Red Group } 30 \\
\text { A }\end{array}$ & $\begin{array}{l}\text { edge } \\
\text { and } \\
\text { top of } \\
\text { petal : } \\
\text { White } \\
\text { Group } \\
155 \mathrm{~A} \text {; } \\
\text { Middle } \\
\text { of } \\
\text { petal: } \\
\text { Red } \\
\text { Purple } \\
\text { Group } \\
\text { N } 66 \mathrm{~A}\end{array}$ & $\begin{array}{l}\text { Red } \\
\text { Purple } \\
\text { Group } \\
58 D\end{array}$ & $\begin{array}{l}\text { Red } \\
\text { Purple } \\
\text { Group } \\
\text { N } 66 \text { A }\end{array}$ & $\begin{array}{l}\text { edge } \\
\text { and top } \\
\text { of } \\
\text { petal: } \\
\text { Orange } \\
\text { Group } \\
28 \mathrm{~A} \\
\text { Middle } \\
\text { of } \\
\text { petal: } \\
\text { Yellow } \\
\text { Orange } \\
\text { Group } \\
17 \text { C }\end{array}$ & $\begin{array}{l}\text { Yellow } \\
\text { group } 13 \\
\text { A }\end{array}$ & $\begin{array}{l}\text { Orange } \\
\text { Red } \\
\text { Group } \\
N 30 B\end{array}$ \\
\hline 8 & $\begin{array}{l}\text { Outer ray } \\
\text { floret : } \\
\text { color } \\
\text { number }\end{array}$ & 1 & 1 & 1 & 2 & 2 & 1 & 1 & 2 & 1 & 1 \\
\hline 9 & $\begin{array}{l}\text { Dark disc } \\
\text { (before } \\
\text { opening of } \\
\text { disc floret) }\end{array}$ & present & present & present & absent & present & absent & present & absent & present & present \\
\hline 10 & $\begin{array}{l}\text { Main color } \\
\text { of perianth } \\
\text { lobes at disc } \\
\text { floret of } \\
\text { outer rows }\end{array}$ & Pink & white & white & Orange & white & Pink & Pink & absent & yellow & Orange \\
\hline 11 & $\begin{array}{l}\text { Pappus: } \\
\text { color of top } \\
\text { relative to } \\
\text { other parts }\end{array}$ & darker & darker & darker & same & darker & same & darker & same & darker & darker \\
\hline
\end{tabular}

Note: $\mathrm{ATH}=$ Athalia, $\mathrm{ZSO}=$ Zsofia, AYU $=$ Ayudia, $\mathrm{NEO}=$ Neoma, NAS $=$ Nashita, NIR $=$ Nirwasita, ARK $=$ Arkadewi, NAL $=$ Nalini, $\mathrm{KIN}=$ Kinandari, $\mathrm{CAN}=$ Candramaya 
Data of ten gerbera varieties decribed that parameter of characters quantitatives had broad genetic variabilities (more than 2) and their scoring showed high heritability (more than 50\%). There were parameter of flower head diameter $(\mathrm{H}: 71,85 \%)$, diameter of first inner ray florets $(\mathrm{H}$ : $94,63 \%)$, disc floret diameter (H: 77,86\%), outer ray floret length $(\mathrm{H}$ : $82,67 \%)$, and outer ray floret width $(93,88 \%)$. These results were similar with previous research, that flower head diameter, diameter of first inner ray florets, disc floret diameter in $F_{1}$ population crossing between Gerbera Local Bali x Rubby Red had broad genetic variability and high heritability [11]. According these results, it could be effective and efficient for selection catagories [10].

There were variability in eleven qualitative characters for 10 varieties of Gerbera (Table 2). Kumar [12] reported that the essential characters in gerbera were color of ray floret and disc floret, flower head type, flower diameter, peduncle length and diameter.

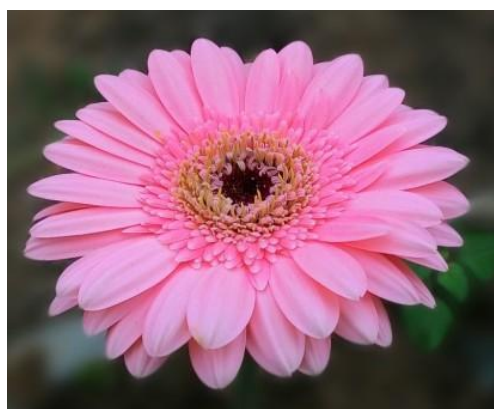

AthaliaAgrihorti

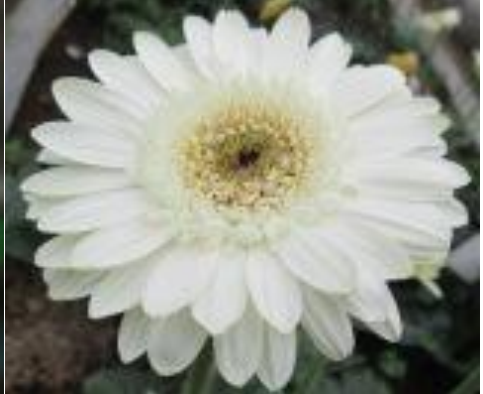

ZsofiaAgrihorti

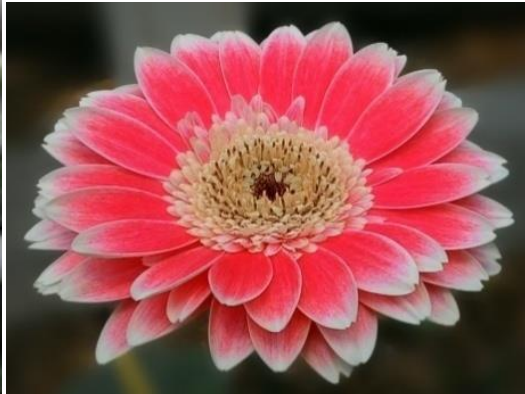

NashitaAgrihorti

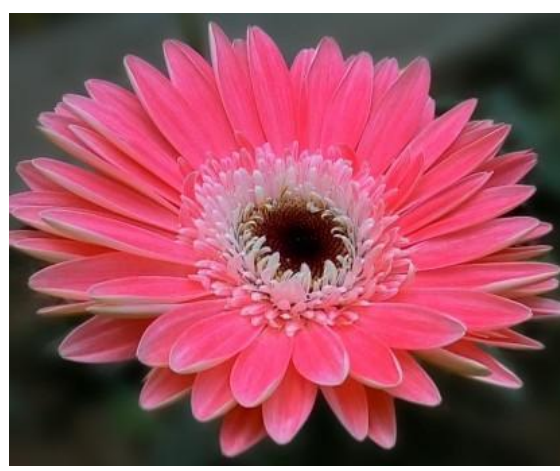

Ayudia Agrihorti

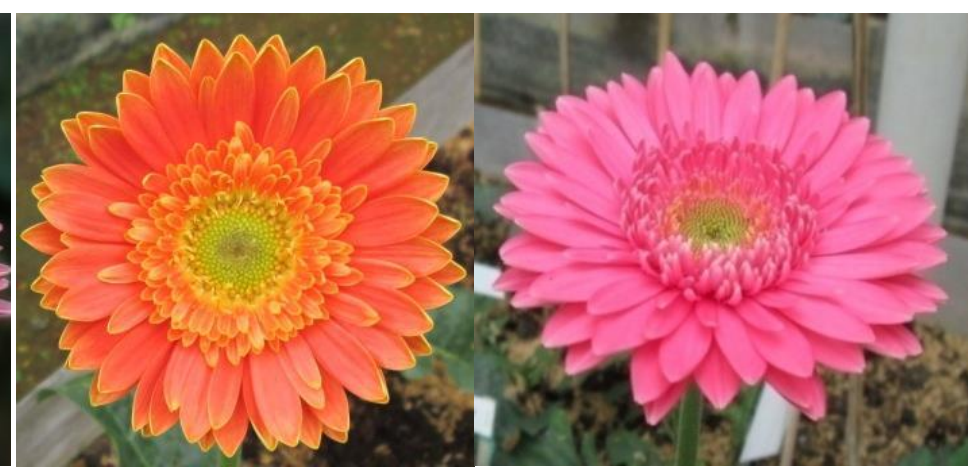

Neoma Agrihorti

Nirwasita Agrihorti

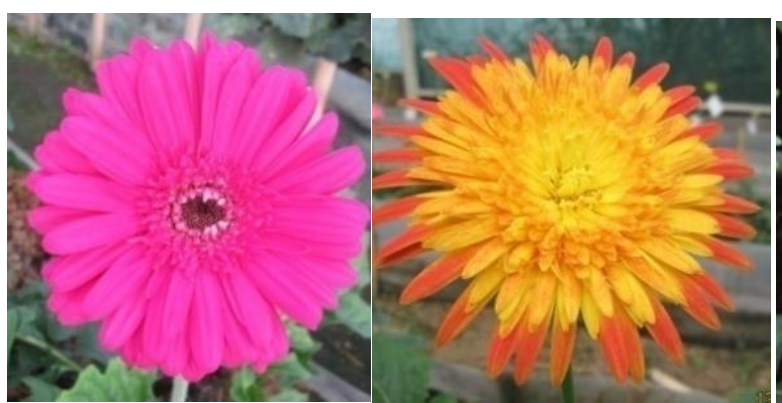

Arkadewi Agrihorti

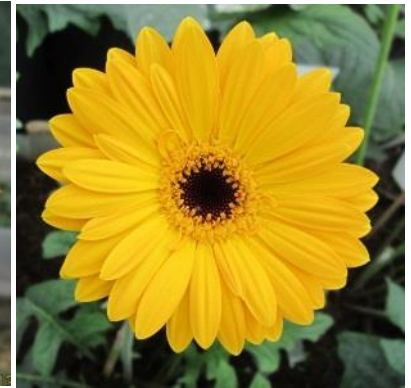

Kinandari Agrihorti

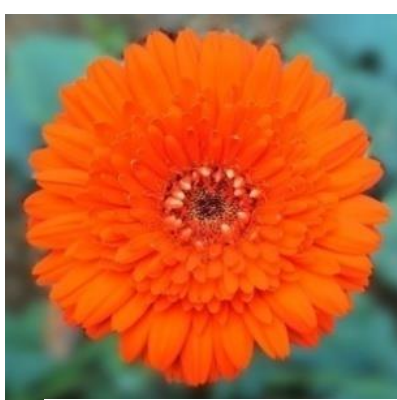

Candramaya Agrihorti

Fig 1. Morphology of ten Gerbera varieties through crossing breeding

The color of ray floret in ten varieties of gerbera observed showed that three of ten ray floret color (red purple) have gradation in color. There was related between anatomy and biochemical characteristic influenced by color in Gerbera [13]. There were most of anthocyanin in pink to red purple Gerbera. Other varieties of Gerbera were white, yellow, orange, orange reddish and red. Main color of perianth lobes at disc floret of outer rows were white (three varieties), pink (three varieties), orange (two varieties) and yellow (one variety). Main color was main value contributor of flower [14]. The different color of Gerbera indicated that there were spectrum color pattern directly showed 
highest morphological level of flower type, flower organ and inner organ [3].

There was variability in disc floret (before opening of disc floret) in ten varieties observed. Seven varieties were dark disc floret and three varieties were bright disc floret, which it was genetically dependence. The ratio of segregation phenotypic indicated that dark disk color was determined by a single dominant gene, designated Dc, and the light disk color by a recessive gene, dc [15].

Types flower head among ten gerbera varieties as described using UPOV Test Guideline [16] is presented in Fig.1. There were single, semi double and double. Eight of ten Gerbera observed were semi double type with two layer of ray floret, one variety (Kinandari Agrihorti) was single type and Nalini Agrihorti was double type.

\section{CONCLUSION}

The broad genetic and phenotypic variabilities and high heritability were obtained for flower head diameter, diameter of first inner ray florets, disc floret diameter, flower head diameter, outer ray floret length and outer ray floret width. There were three kind of flower head type (single, semi double and double). Gerbera Kinandari was single, semi double were Athalia Agrihorti, Zsofia Agrihorti, Ayudia Agrihorti, Neoma Agrihorti, Nashita Agrihorti, Nirwasita Agrihorti, Arkadewi Agrihorti, and Candramaya Agrihorti, while Nalini Agrihorti was double.

\section{REFERENCES}

[1] Nurmalinda and A.Yani, 'Cutting Flower of gerbera Preference', J. Hort, vol. 19, no. 4, pp. 450 - 458, 2009.

[2] Tourjee, K.R., Harding A and Byrne T.G, 'Early Development of Gerbera as a Floricultural Crop', Hort. Tech, vol. 4, no. 1, pp. 34-40, 1994.

[3] Teeri T.H., Elomaa P., Kotilainen M., Albert V.A, 'Mining plant diversity: Gerbera as a model system for plant developmental and biosynthetic research', BioEssays, vol 28, pp. 756-767, 2006.

[4] Anand, M., A. Sankari and K. Nageswari, 'Evaluation of genotypes of gerbera (Gerbera jamesonii Bolus ex. Hooker F.) under open condition at Yercaud', International Journal of Agricultural Sciences. Vol. 10, no. 2, pp. 498 505, 2014.

[5] Lifia Y. O. and R. Hariance, 'Bauran pemasaran bunga gerbera (Gerbera jamesonii) di kelompok tani Boemi Nursery Jawa Barat', Journal of Agribusiness and Community Empowerment. vol. 1 , no. 1 , pp. $38-48,2018$.

[6] Kementerian Pertanian, 'Basis data statistik pertanian',

http://aplikasi2.pertanian.go.id/bdsp/id/komodita s. Diakses tanggal 7 Mei 2020.

[7] Wahdah, R., A. Baihaki, R. Setiamihardja and G. Suryatmana, 'Variabilitas dan heritabilitas laju akumulasi bahan kering pada biji kedelai'. Zuriat, Vol. 7. No. 2, pp. 92-98, 1996.

[8] Fehr, W. R, 'Principles of Cultivar Development', Macmillan Publishing Company, New York, pp. 536, 1987.

[9] Mangoendidjojo, W, 'Dasar-dasar Pemuliaan Tanaman, Penerbit Kanisius. Yogyakarta. Pp. 182, 2003.

[10] Pinaria, A., A. Baihaki, R. Setiamihardja and A. Darajat, "Variabilitas genetik dan heritabilitas karakter-karakter biomasa 53 genotip kedelai', Zuriat, Vol. 6 No. 2, pp. 88-92, 1995.

[11] Yuniarto, K., R. Kurniati, Suryawati and R. Meilasari, 'The phenotypic performances of Gerbera Local Bali x Rubby Red hybrids', Agrivita Journal of Agricultural Sciences, vol. 40, no. 1, pp. 8 - 14, 2018.

[12] Kumar, R, 'Evaluation of gerbera (Gerbera jamesonii Bolus ex. Hooker F.) genotypes for flower quality traits under naturally ventilated polyhouse', TAJH, vol. 8, no. 2, pp. 680-682, 2013.

[13] Hatamzadeh, A., Akbari, R., Sariri, R., and Bakhsi, D, Comparison of Parameters affecting flower color in Gerbera hybrid: A phytochemical study on new varieties, Journal of Agricultural Science, vol. 4, no.11, 2012.

[14] Oud, J. S. N., Harrie Schneiders, Ad J. Kool and Mart Q. J. M. van Grinsven, 'Breeding of transgenic orange Petunia hybrid varieties', Euphytica, vol. 84, pp. 175-181, 1995.

[15] Kloos, W.E., George C.G., and Sorge L.K, 'Dark disk color in the flower of gerbera hybrid is determined by a dominat gene', Dc. Hortscience vol. 40, no. 7, pp. 1992-1994, 2005.

[16] UPOV,.' Guidelines for The Conduct of Test for Distinctness, Uniformity and Stability. Gerbera (Gerbera Cass.)', International Union for the Protection of New Varieties of Plants, TG/77/9. Geneva, 2000. 